C.J.G. Plummer

R. Gensler

H.H. Kausch

\section{Lattice imaging in melt crystallized polypropylene thin films}

Received: 27 June 1997

Accepted: 15 August 1997
Dr. Christopher J.G. Plummer ( $\square)$ Rudolf Gensler - Hans-Henning Kausch École Polytechnique Fédérale de Lausanne Département des Matériaux-Laboratoire de Polymères

MX-D Ecublens

CH-1015 Lausanne/Switzerland

Tel: + 41216932847 Fax: + 41216935868

\begin{abstract}
Melt crystallized isotactic polypropylene thin films of thickness between 30 and $100 \mathrm{~nm}$ have been investigated by high-resolution transmission electron microscopy at room temperature. The $c$-axis projection of the $2 * 3_{1}$ helices and their packing in the lattice were clearly visible in flat-on lamellae of the $\alpha$-phase following reconstruction from the components of the image Fourier transform corresponding to the $\left(\begin{array}{lll}1 & 1 & 0\end{array}\right)$ and $(040)$ lattice planes, and the image power spectra also indicated contributions from (130) and $(060)$ relfections, corresponding
\end{abstract}

to a line resolution of about $0.35 \mathrm{~nm}$. These results are discussed in terms of Bloch wave calculations based on the generally accepted structure for the $\alpha$-phase. Attempts to obtain lattice images of the $\beta$-phase in isotactic polypropylene and melt crystallized syndiotactic polypropylene under similar operating conditions are also briefly discussed, although these provided relatively little structural information.

Key words TEM - HREM - isotactic polypropylene - syndiotactic polypropylene - image simulation

\section{Introduction}

In recent years, there has been considerable use of highresolution transmission electron microscopy (HREM) to investigate chain packing in semicrystalline polymers [1-5]. Initially, HREM studies were restricted to electron beam resistant polymers, but it has since been demonstrated that the investigation of relatively beam sensitive polymers such as polyethylene [6], poly(tetrafluoroethylene) $[7,8]$, poly(hydroxybutyrate) $[9]$ and cellulose $[10]$ is also feasible, given suitable precautions against radiation damage. To our knowledge, HREM studies of polypropylene have not so far been reported in the literature, although its crystal architecture has been extensively investigated in the past using scattering techniques [11-21], and there continues to be considerable interest in specific details of the structure of the various polymorphic forms of both isotactic polypropylene (iPP) and syndiotactic polypropylene (sPP) [22-28]. Direct lattice imaging of iPP has been shown to be possible using atomic force microscopy (AFM) [29-33], but HREM may prove to be a useful complementary technique in so far as it provides information on the internal, possibly defective structure of a sample, whereas AFM is restricted to investigations of the surface morphology.

In what follows we present the results of an HREM investigation of thin melt crystallized polypropylene films, with emphasis on the iPP monoclinic $\alpha$-phase. iPP can crystallize in at least two other forms, namely the hexagonal $\beta$-phase and the triclinic $\gamma$-phase $[12,16,28]$. Subsequent to rapid cooling or cold drawing, a fourth modification, known as the mesomorphic or smectic phase is also observed, although the details of its crystalline structure are less well known $[24,25]$. The $\alpha$-modification is the most usual form under normal processing 
(a)
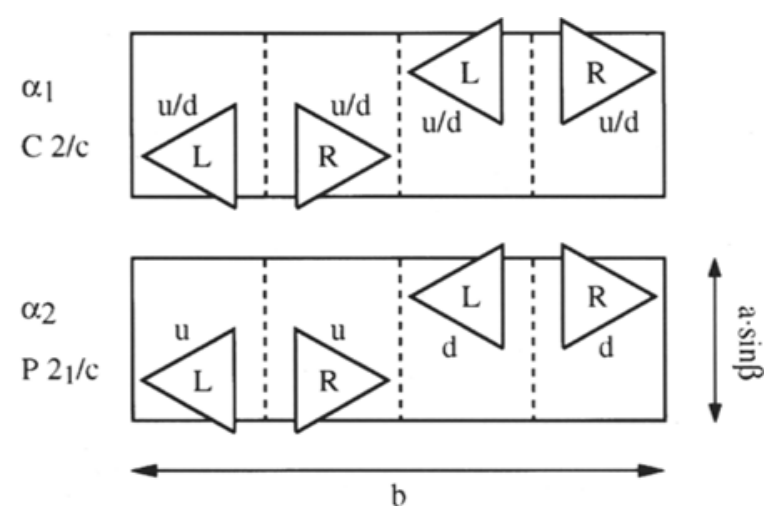

(b)

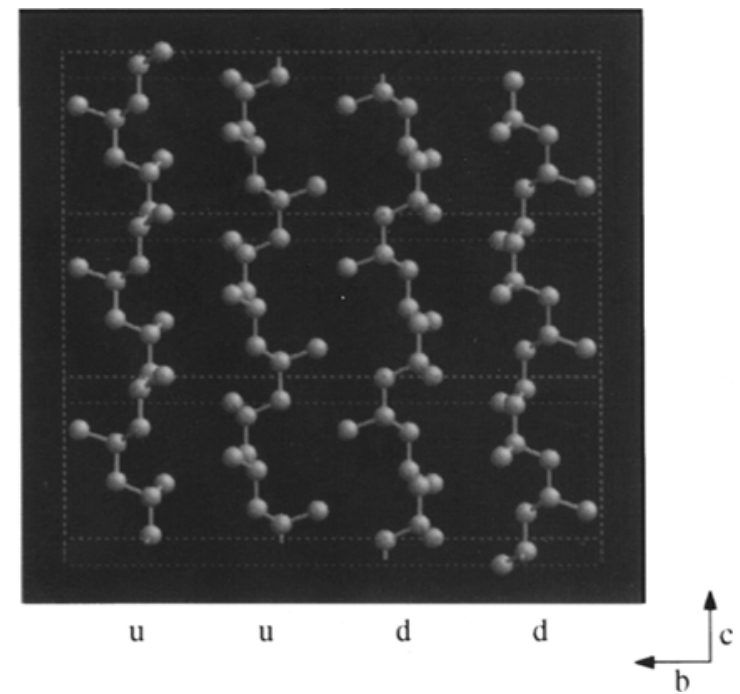

Fig. 1 (a) The structure of $x$-iPP (unit cell viewed along the $c$-axis): the two modifications differ in the arrangement of "up" (u) and "down" (d) helices; " $L$ " and " $R$ " indicate left- and right-handed helices; (b) three unit cells of the $\alpha_{2}$-form viewed perpendicular to the chain axis (for clarity the hydrogen atoms are not shown)

conditions. Individual $\beta$-iPP spherulites also occur sporadically, however, and their formation can be promoted by adding $\beta$-nucleants [34].

In each case the iPP chains are packed in the lattice in the form of right- or left-handed $2 * 3_{1}$ helices. The helices may further be classified as "up" and "down" helices depending on the orientation of the methyl side groups with respect to the chain axis. Extensive $X$-ray studies $[13,14,18,19]$ have revealed two forms of the monoclinic $\alpha$-phase, which differ in the configurational arrangement of the helices in the unit cell as shown schematically in Fig. 1a. The $\alpha_{1}$-modification shows random packing of "up" and "down" helices (space group C2/c) and is formed during melt crystallization at high undercoolings $[13$, $14,21]$. In the more stable $\alpha_{2}$-modification, observed at low undercoolings or after annealing at elevated temperatures $[18,19,21]$, the helices are packed in alternating layers of "up" and "down" helices (space group $\mathrm{P} 2 / \mathrm{c}$ ) $[13,14]$. This is illustrated in Fig. 1b where three unit cells of the $\alpha_{2}$-modification are shown projected along $a^{*}$. The arrangement of helices of a given handedness is nevertheless the same in the two modifications, as shown in Fig. 1a, and there are only marginal differences in the unit cell parameters (the literature values are generally in the range $a=0.661-0.666 \mathrm{~nm}, b=2.073-2.098 \mathrm{~nm}$, $c=0.6495-0.653 \mathrm{~nm}$ and $\beta=98.5^{\circ}-99.62^{\circ}[11-15]$ ).

\section{Experimental}

The iPP investigated here was an additive free homopolymer kindly supplied by PCD Polymere, Austria. The weight average molecular weight, $M_{\mathrm{w}}$, was determined by gel permeation chromatography to be about $300000 \mathrm{~g} \mathrm{~mol}^{-1}$ and the polydispersity index was 2.97 . Thin films were prepared by drawing freshly cleaved mica plates from a xylene solution at $140^{\circ} \mathrm{C}$ under nitrogen. AFM indicated the average thickness of the films to vary between 30 and $50 \mathrm{~nm}$, with local thickness variations of about $15-20 \mathrm{~nm}$. To obtain samples with a well defined thermal history, the films were remelted on the mica substrate for $30 \mathrm{~s}$ at $210^{\circ} \mathrm{C}$ on a hot stage, and subsequently isothermally recrystallized at temperatures ranging from 110 to $125^{\circ} \mathrm{C}$, again under nitrogen. They were then floated off the substrate onto a water bath, mounted on carbon coated copper TEM grids and dried. As an alternative, in order to investigate the feasibility of HREM investigation of free standing films, somewhat thicker samples ( $\geq 70 \mathrm{~nm}$, as measured by AFM) were mounted on coarsemesh annealed copper grids of the type used by Lauterwasser and Kramer for TEM investigations of crazing [35]. In this case one grid square spans the whole of the TEM sample holder aperture, so that apart from a thin carbon coating to reduce charging, the film is essentially self-supporting. Although somewhat disadvantageous from the point of view of sample mechanical stability, this method may evantually prove useful for the observation of deformed samples [36]. To reveal any edge-on or close to edge-on lamellar structure, certain samples were stained in $\mathrm{RuO}_{4}$-vapour, the stain being prepared as described by Montezinos et al. [37].

Unless stated otherwise, HREM was carried out at room temperature using the Philips CM 20 (accelerating voltage $200 \mathrm{kV}, C_{\mathrm{S}}=2.0 \mathrm{~mm}$, point resolution $0.28 \mathrm{~nm}$ ), equipped with a low-dose unit. No objective aperture was used. For $\alpha$-iPP, the plate magnification in the FOCUS and EXPOSURE mode was generally set to $38000 \times$, and the exposure times were chosen to be within the total lifetime of the (130) diffraction spots for the same condenser settings (depending on the film thickness the exposure 
times at this magnification were at most one third of those given by the automatic exposure meter). Since we worked without an objective aperture and at relatively low magnifications, it was often difficult to identify the point of Gaussian focus to within more than about $5 \mathrm{~nm}$ (although the presence of small dust particles on the films was helpful in this respect). Once Gaussian focus was found, the image was defocussed by -70 to $-80 \mathrm{~nm}$ using the objective lens fine focus control, in order to obtain Scherzer or "extended" Scherzer focus. Again, because of the low magnifications used in this study, the amount of defocus could not be determined a posteori from the experimental images so that the levels of defocus quoted in what follows are subject to an estimated error of $\pm 5 \mathrm{~nm}$. The microscope was not calibrated for the determination of absolute values of the total end point dose, but the beam sensitivity was similar to that of polyethylene. For comparison, Revol and Manley [6] have obtained $0.37 \mathrm{~nm}$ resolution lattice images of polyethylene single crystals at $120 \mathrm{kV}$ and at a magnification of $36000 \times$ with a total dose of $1.8 \times 10^{-11} \mathrm{C}_{\mu \mathrm{m}^{-2}}$.

After the first exposure, a second image was recorded in the SEARCH mode at a plate magnification of $11500 \times$ and at high defocus in order to situate the initial micrograph with respect to any coarse features of the sample. The images were recorded on Kodak SO-163 photographic film, developed in full strength Kodak developer D-19 for $15 \mathrm{~min}$. The negatives were then examined with an optical microscope. Regions of interest were digitized using a $\mathrm{CCD}$ camera and transferred to a computer for image analysis. For the simulation of HREM images Bloch wave calculations were carried out using the Electron Microscopy Simulation (EMS) software package [38]. Some complementary multislice simulations were also performed using the Cerius ${ }^{2}$ package from Molecular Simulations Inc.

\section{Results and discussion}

The overall microstructure of a stained $30 \mathrm{~nm}$ thick iPP film, which was melt crystallized at $120^{\circ} \mathrm{C}$, is shown at low magnification in Fig. 2a. In all the samples a two-dimensional spherulite-like morphology was observed, with spherulite radii of the order of $10 \mu \mathrm{m}$. Because of the limited film thickness and the presence of the substrate during crystallization however, the lamellar orientation in these spherulites was expected to be modified with respect to that in thicker spherulitic samples crystallized under equivalent conditions, in which a characteristic crosshatched arrangement of lath-like lamellae is observed [39]. When stained, the thinnest films appeared relatively featureless at the submicron level at high defocus and
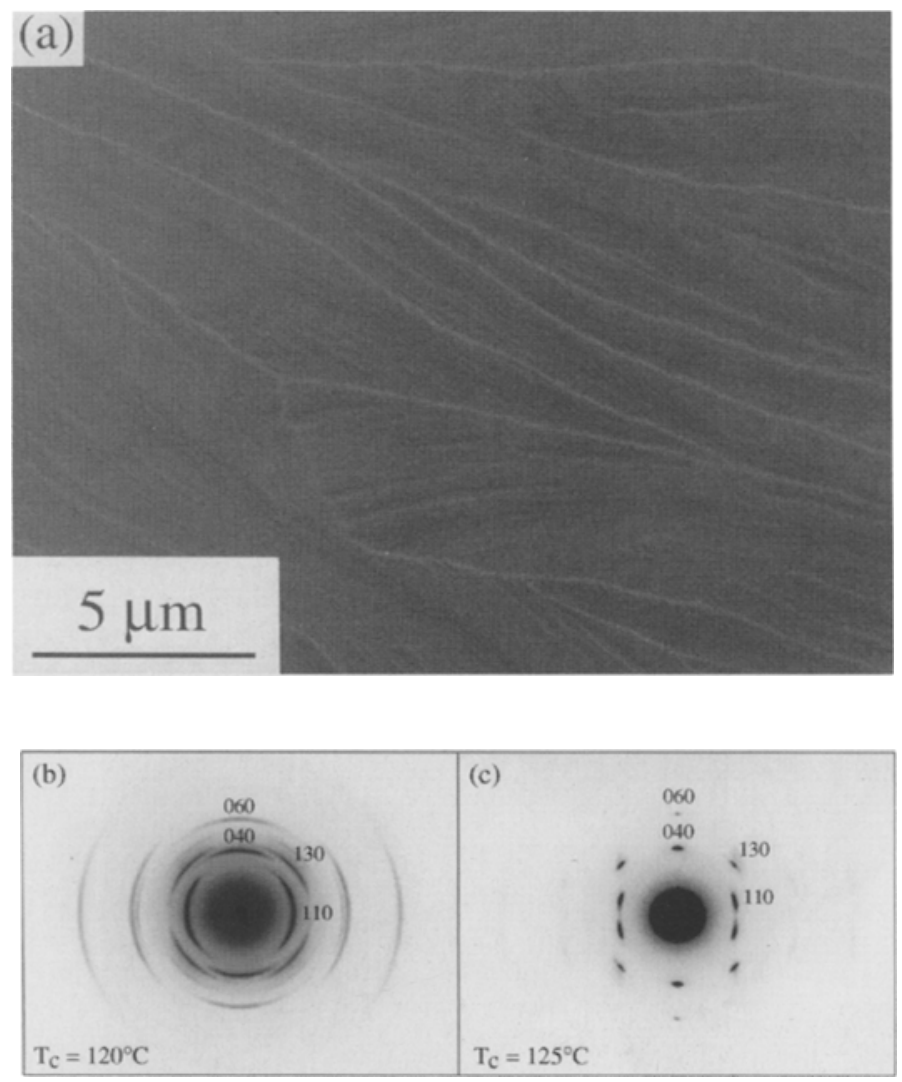

Fig. 2 (a) Microstructure of a thin film isothermally crystallized at $120^{\circ} \mathrm{C}$, and electron diffraction patterns of samples crystallized at (b) $120^{\circ} \mathrm{C}$ and (c) $125^{\circ} \mathrm{C}$

selected area electron diffraction (SAED) suggested a predominantly flat-on arrangement of the lamellae (Fig. 2b). The coarse microstructure changed little with crystallization temperature in the range $110-125^{\circ} \mathrm{C}$, although sharpening of the SAED diffraction spots for a given selected area aperture size suggested there to be an increase in spatial coherence with increasing crystallization temperature (compare, e.g., Fig. 2b and c).

Figure 3 shows a second image of a sample crystallized at $120^{\circ} \mathrm{C}$, taken immediately after an initial exposure at high magnification. The dark circular region resulted from beam damage during focusing, and the light streaks, thought to result from shrinkage during crystallization, lie along the spherulite radii. Part of the HREM lattice image recorded at about $-75 \mathrm{~nm}$ defocus is shown in Fig. 4, corresponding to the centre of the region shown in Fig. 3. The image is noisy, reflecting the low exposure times and the granularity of the photographic emulsion, as well as the presence of amorphous material in the path of the beam. Nevertheless, two sets of lattice fringes are clearly visible within an irregularly shaped patch whose total area was about $35 \times 35 \mathrm{~nm}^{2}$. Figure $5 \mathrm{a}$ shows the power 


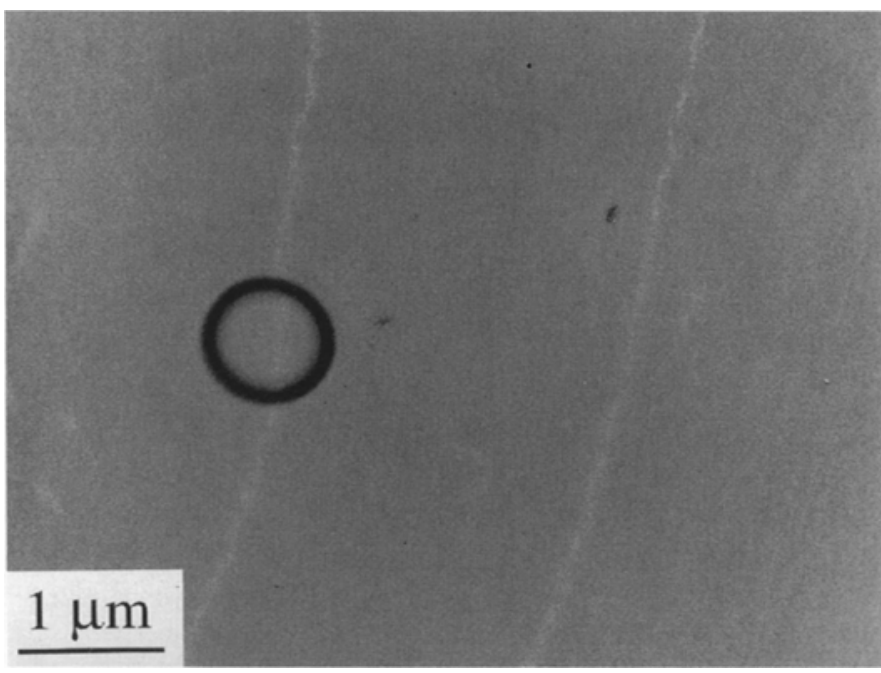

Fig. 3 Typical "second image" taken immediately after initial exposure of a film isothermally crystallized at $120^{\circ} \mathrm{C}$. The dark circular region corresponds to the part of the film used for focusing and the HREM image came from the centre of the region shown in the micrograph

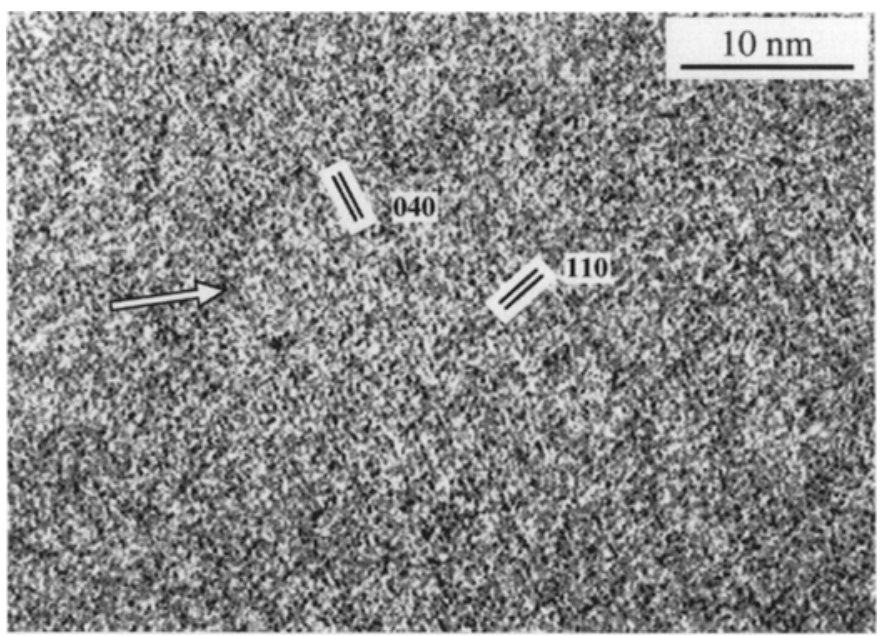

Fig. 4 Lattice image in a thin film of iPP isothermally crystallized at $120^{\circ} \mathrm{C}$ obtained at about $-75 \mathrm{~nm}$ defocus

spectrum obtained form a two dimensional Fast Fourier Transform (FFT) of a $256 \times 256$ pixel image taken from the centre of Fig. 4 (Fig. 5 is in the same orientation as Fig. 4). Comparison of the relative orientation of the fringes visible in Fig. 4 with the positions of the peaks in the power spectrum in Fig. 5a, and the experimental diffraction patterns shown in Figs. $2 b$ and $2 c$, indicates the fringes in Fig. 4 to correspond to the $(040)$ and $(110)$ planes in the $\alpha$-iPP unit cell. By tilting the Fig. 4 one can also

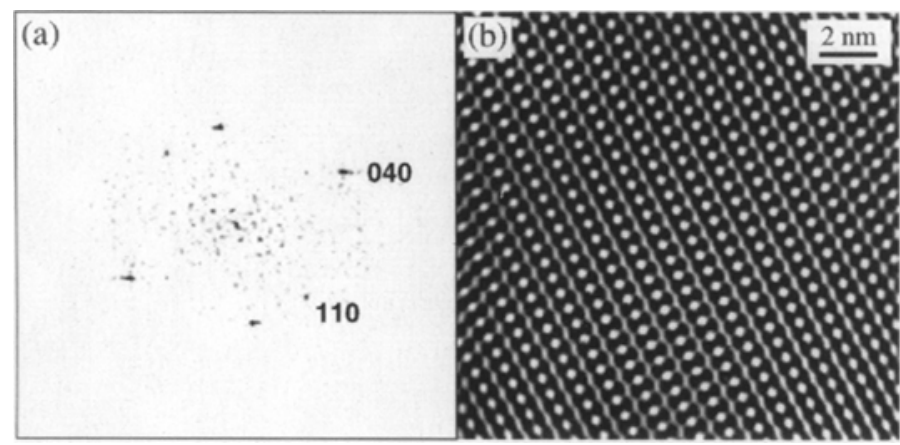

Fig. 5 (a) FFT power spectrum of part of the lattice image shown in Fig. 4; (b) lattice image reconstructed by Fourier filtering

distinguish a set of ( 110$)$ fringes (the appropriate viewing direction is indicated by the arrow). The corresponding spacings are $d_{110}=0.626 \mathrm{~nm}$ and $d_{040}=0.52 \mathrm{~nm}$ which are well within the resolution limit of the CM 20, assuming ideal operating conditions.

Fourier filtering was carried out either by masking out the noise and back transforming, or indirectly by masking the periodic component of the FFT, back transforming and subtracting the resulting image from the original image. The result of filtering is shown in Fig. $5 \mathrm{~b}$ and a further enlargement is given in Fig. 6 along with the projection of the unit cell along the $\left[\begin{array}{ll}0 & 0\end{array}\right]$ direction. Assuming a lamellar thickness of the order of $10 \mathrm{~nm}$, and correct focussing, it is reasonable to take these images to represent an approximation to the projected potential of the lattice [40], in which case the dark regions correspond to the projection of the iPP helices along [0 011$]$. The periodic arrangement is clearly consistent with that shown in Fig. 1, although the resolution is insufficient to reveal the pitch of the individual helices.

As an aid to more detailed interpretation and in order to identify the optimum operating conditions for lattice imaging. Bloch wave calculations were carried out using the EMS software package [38] (the helical direction

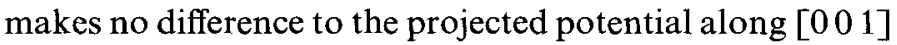
and so, for convenience, the calculations referred to here are for the $\alpha_{2}$-modification). To begin with, we examined the influence of sample thickness $(t)$ and defocus $(\Delta f)$ with the objective aperture diameter $(\varnothing)$ set to $7 \mathrm{~nm}^{-1}$ which corresponds approximately to the resolution limit of the $\mathrm{CM}$ 20. Scherzer focus for this microscope $\left(\Delta f=-\left(C_{\mathrm{S}} \lambda\right)^{1 / 2}\right)[40]$ occurs at $\Delta f=-71 \mathrm{~nm}$, whereas the experimental HREM images were recorded under "extended Scherzer" conditions, that is, for somewhat higher values than $-71 \mathrm{~nm}$ (typically $\Delta f=-75$ to $-80 \mathrm{~nm}$ ). Figure 7 shows simulated HREM images for five $\Delta f(-70$ to $-90 \mathrm{~nm})$ and four $t(5-20 \mathrm{~nm})$. For $t=5-10 \mathrm{~nm}$ and $\Delta f=-70$ to $-90 \mathrm{~nm}$ the images reflected well the threefold symmetry 


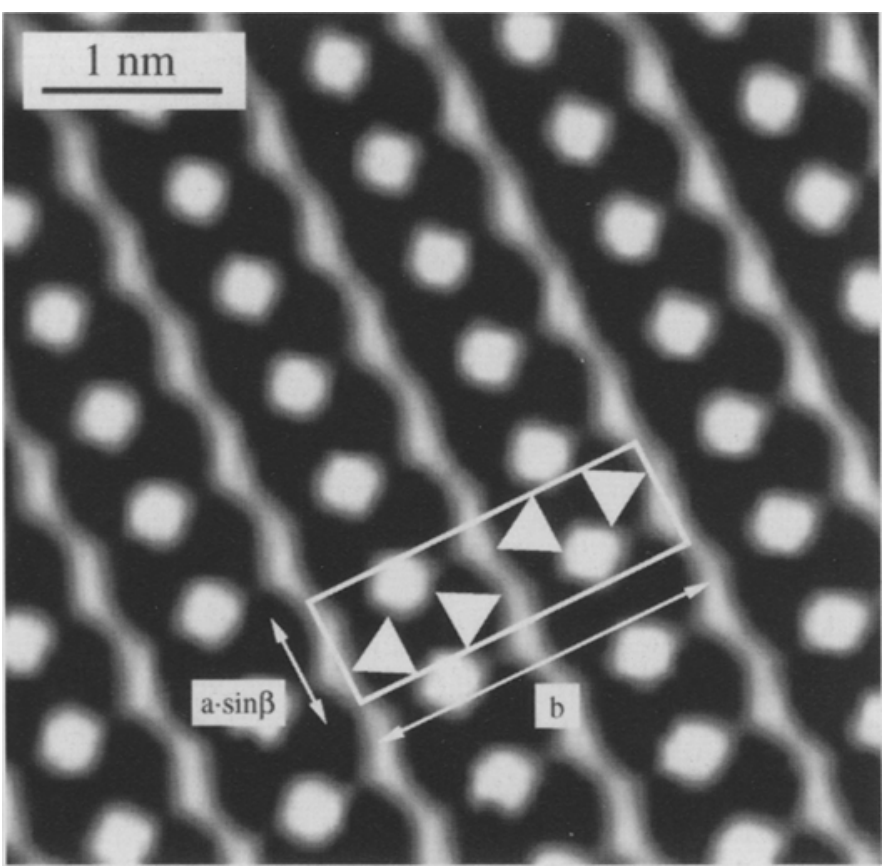

Fig. 6 Enlargement of Fig. 5b with the $c$-axis projection of the unit cell as indicated

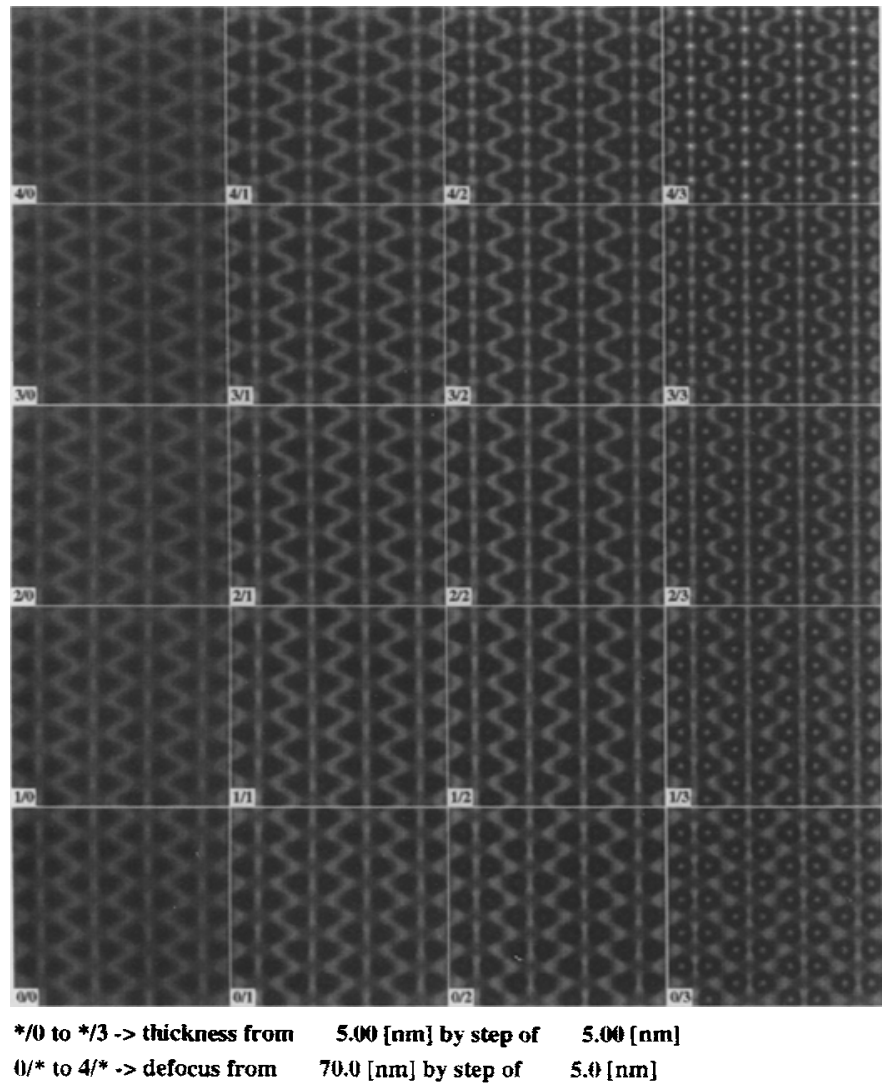

Fig. 7 Simulated HREM images of $\alpha$-iPP in the $c$-axis projection as a function of defocus $(\Delta f)$ and sample thickness $(t)$ of the helices, and the projected potential approximation is clearly valid in this range of thicknesses, as anticipated above. The images which correspond most closely to the projected potential were obtained for $t=10 \mathrm{~nm}$ and $\Delta f=-75 \pm 5 \mathrm{~nm}$ (images $0 / 1$ to $2 / 1$ in Fig. 7), suggesting the experimental conditions to be optimal, assuming accurate alignment of the microscope and the sample, accurate defocusing, zero objective astigmatism and negligible beam damage and noise levels. For $t \geq 15 \mathrm{~nm}$ and $\Delta f<-75 \mathrm{~nm}$ the beginning of contrast reversal can be seen in Fig. 7. Complete contrast reversal was found to occur at $t=30-35 \mathrm{~nm}$ (the corresponding simulated images are not shown in Fig. 7). However, this is well beyond the expected lamellar thickness of about $10 \mathrm{~nm}$ in iPP samples melt crystallized in the temperature range considered here $\left(110-125^{\circ} \mathrm{C}\right)$.

Typical experimental lattice images of samples crystallized at $120^{\circ} \mathrm{C}$, such as that in Fig. 4, did not show resolution to $0.28 \mathrm{~nm}$, the $(130)$ and $(060)$ reflections being absent, for example. To make comparison meaningful, it was therefore necessary to reduce the effective objective aperture diameter in the simulations to between $\varnothing=2\left(d_{040}\right)^{-1}=2(0.52 \mathrm{~nm})^{-1}=3.85 \mathrm{~nm}^{-1}$ and $\varnothing=$ $2\left(d_{130}\right)^{-1}=2(0.4765 \mathrm{~nm})^{-1}=4.2 \mathrm{~nm}^{-1}$. Figure 8 shows a simulated lattice image for $\Delta f=-75 \mathrm{~nm}$ and $t=10 \mathrm{~nm}$

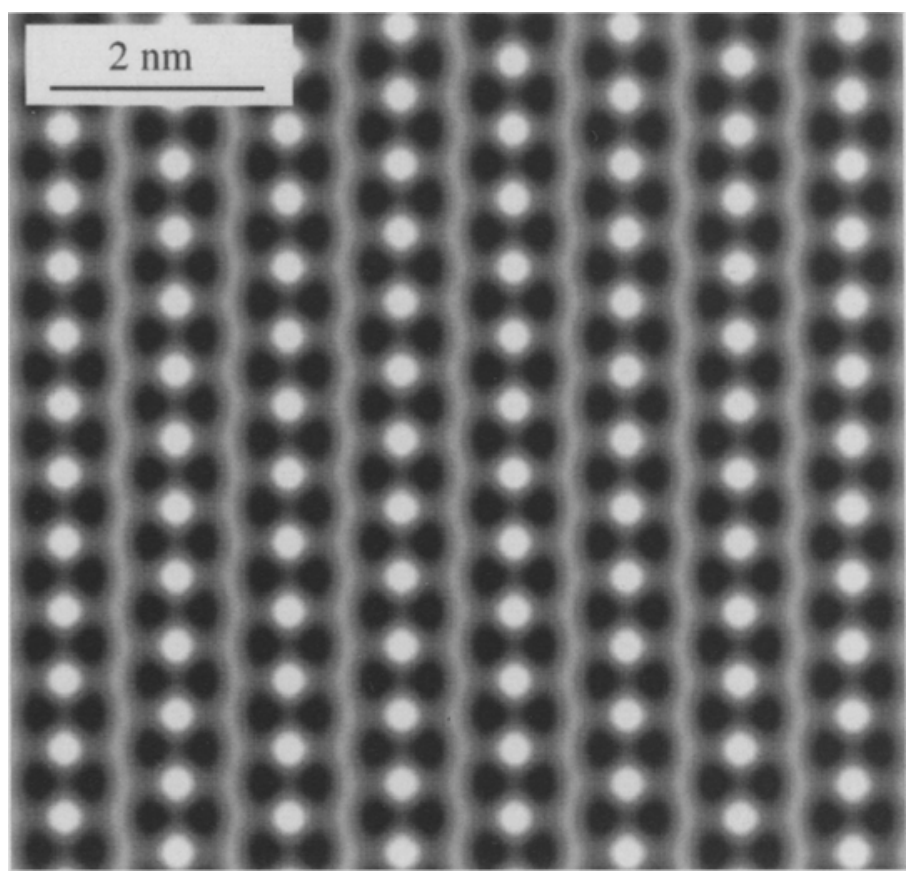

Fig. 8 Simulated HREM image of $\alpha$-iPP in the $c$-axis projection for $\Delta f=-75 \mathrm{~nm}$ and $t=10 \mathrm{~nm}$; the diameter of the objective aperture was reduced to $\varnothing=4 \mathrm{~nm}^{-1}$ to avoid the transmission of the (1 30$)$ beams 
where $\varnothing$ was set to $4 \mathrm{~nm}^{-1}$, giving satisfactory agreement with Fig. 6. The absence of $\left(\begin{array}{ll}3 & 30)\end{array}\right)$ and $(060)$ reflections was therefore tentatively attributed to loss of near field order in the lattice owing to beam damage.

In HREM images of samples crystallized at $125^{\circ} \mathrm{C}$, where the degree of local order and/or beam resistance may have increased somewhat, it was nevertheless possible to identify regions such as shown in Fig. 9, for which the image power spectrum did suggest a significant contribution from the (130) planes. Both the image and the power spectrum have been contrast enhanced for reproduction, but the signal-to-noise ratio in the original image was sufficient to permit Fourier filtering, the results of which are also shown. The agreement with simulations in which the aperture diameter was adjusted to admit the (130) beams was less good than in the previous example, although the filtered image in Fig. 9c does provide a more accurate representation of the projected potential of the ideal structure. Finally, Fig. 10 shows one of several images taken at a somewhat lower dose than in Fig. 9, apparently containing contributions from $(060)\left(d_{060}=\right.$ $0.347 \mathrm{~nm}$ ), but in such cases the noise level was too high for simple Fourier filtering to give meaningful results.

It is of course possible to use diffraction data to correct the positions and amplitudes of the Fourier components, assuming the observed phases to be approximately correct, and indeed one can deduce the phases of, and insert reflections which are present in the diffraction pattern, but absent from the image power spectrum [41]. However, this is tantamount to doing electron crystallography on what is already considered to be a known structure. It also presupposes a high degree of periodicity, whereas one of the potential uses of HREM in polymers is for the observation of lattice defects, for which Fourier filtering is less appropriate $[42,43]$. Indeed we think that defects do contribute substantially to the "noise" in images such as that in Fig. 10, where there is discernible streaking of the $(110)$ peaks in the power spectrum. If one looks at the image at an oblique angle the $\left(\begin{array}{lll}1 & 1 & 0\end{array}\right)$ planes a loss in crystallographic registry is apparent on moving across the micrograph (a clearer example of a defective structure is given below).

For TEM of the thicker films mounted on coarse copper grids, use was made of the Philips EM 430 ST operated at $300 \mathrm{kV}$ (point resolution $0.2 \mathrm{~nm}, C_{\mathrm{S}}=$ $1.15 \mathrm{~mm}$ ). Very similar results to those described above were obtained for the thin films using this microscope under similar operating conditions, and no additional reflections were detected in the image power spectra. The EM 430 ST was nevertheless preferred for films with a nominal thickness of the order of $70 \mathrm{~nm}$ owing to the somewhat greater transmission at $300 \mathrm{kV}$ (although AFM suggested the local thickness of the films to vary by about
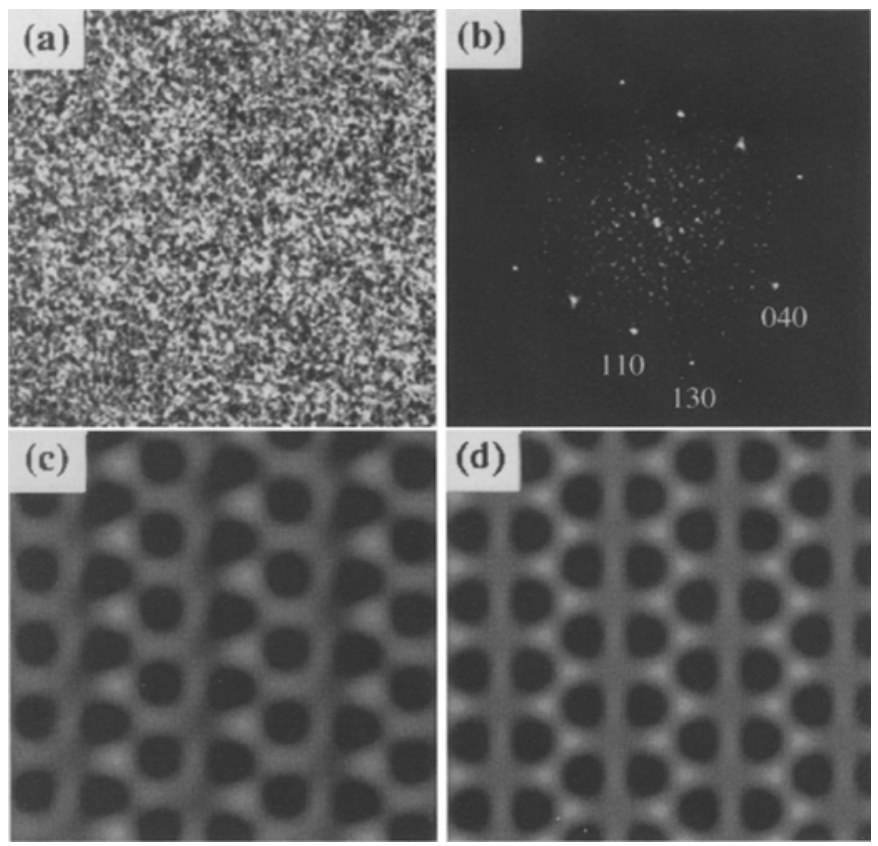

Fig. 9 (a) Lattice image of a thin film of iPP isothermally crystallized at $125^{\circ} \mathrm{C}$; (b) FFT power spectrum of (a) showing (1 30 ) reflections; (c) detail of the corresponding Fourier filtered image; (d) simulated image
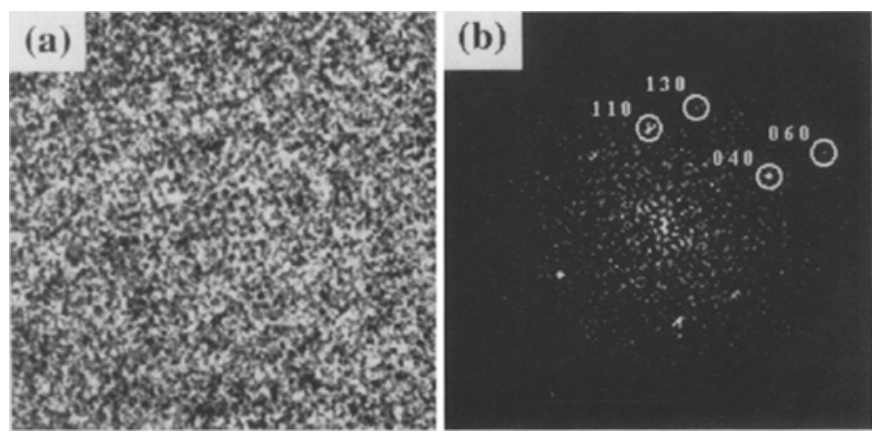

Fig. 10 Lattice image of a thin film of iPP isothermally crystallized at $125^{\circ} \mathrm{C}$, and the corresponding FFT power spectrum showing $(060)$ reflections

$\pm 20 \mathrm{~nm}$, so that individual images did not necessarily correspond to a total thickness of $70 \mathrm{~nm}$ ). Figure 11 shows a low magnification, highly defocused image of a stained ca. $70 \mathrm{~nm}$ thick film crystallized at $110^{\circ} \mathrm{C}$, showing the cross-hatched lamellar structure in part a 2-D spherulite. SAED nevertheless indicated the presence of a substantial proportion of flat-on lamellae, which may reflect the influence of the film-substrate interface. Indeed most of the lattice images obtained from these samples resembled Fig. 4, showing irregular patches of fringes with [ $\left[\begin{array}{lll}0 & 0 & 1\end{array}\right]$ 
Fig. 11 The cross-hatched lamellar structure of a $70 \mathrm{~nm}$ thick iPP film crystallized at $110^{\circ} \mathrm{C}$ and stained with $\mathrm{RuO}_{4}$ vapour

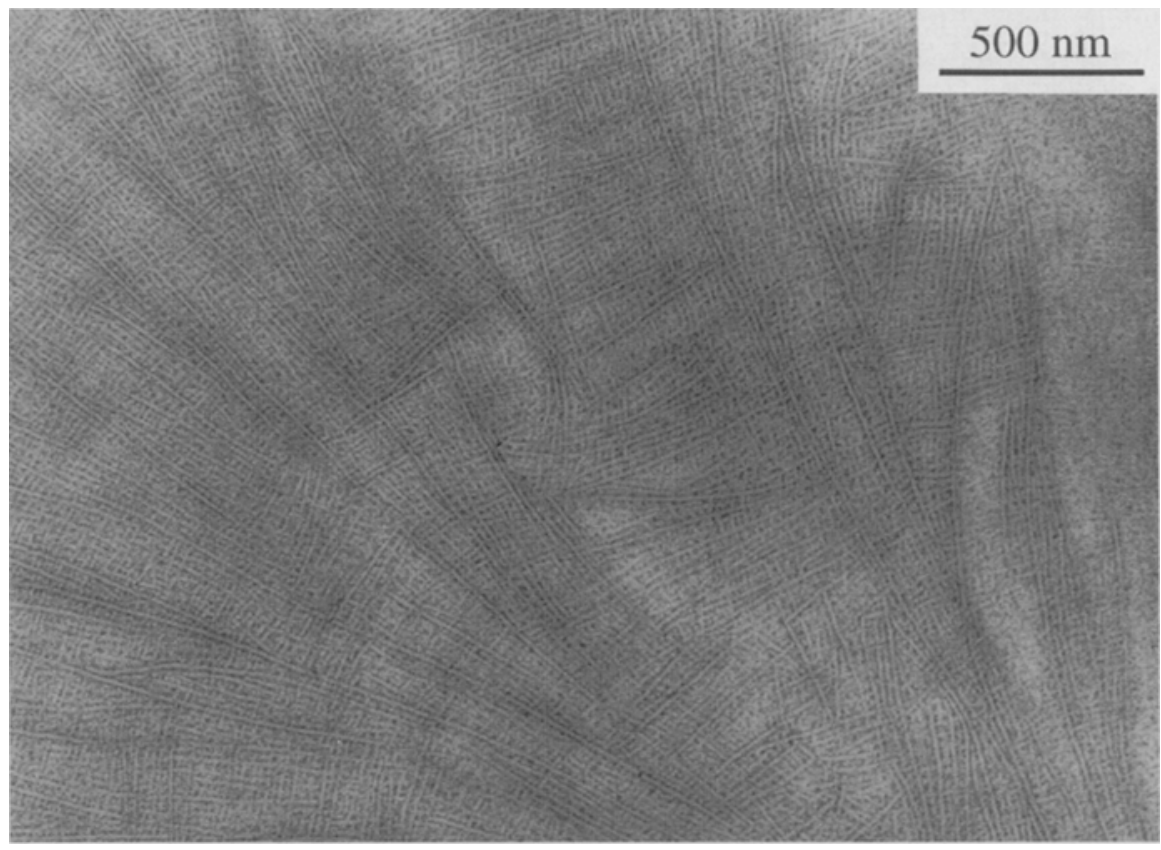

vertical (dark field micrographs obtained using the (h $\mathrm{k} 0$ ) reflections also suggested the absence of a regular habit for lamellae in this orientation). No other crystallographic orientations could be identified directly from the HREM micrographs, and no images were obtained for lattice planes other than those belonging to the [ $\left[\begin{array}{lll}0 & 0 & 1\end{array}\right]$ zone.

Many of the images nevertheless showed a distinct morphology consisting of bands of fringes of between 10 and $20 \mathrm{~nm}$ in width and up to $100 \mathrm{~nm}$ or more in length. These were taken to reflect a lath-like lamellar habit. An example is shown in Fig. 12, taken from a sample crystallized at $110^{\circ} \mathrm{C}$, where one set of $(110)$ fringes is visible. It was not possible to identify the lamellar orientation in this case, but if its long axis is taken to be parallel to $a^{*}$, the lamellar growth direction, this lamella must be nearly flat-on. It was therefore inferred that these films contained lath like lamellae with a wide range of orientations, superimposed on a layer of irregularly shaped flat-on lamellae which was in contact with the substrate during crystallization. Of additional interest in Fig. 12 is the apparent presence of an array of crystallographic defects. The position of an additional $\left(\begin{array}{lll}1 & 1 & 0\end{array}\right)$ half plane is indicated by the arrow, for example (again, tilting the figure helps to see this more clearly). The existence of edge dislocations of this type may be anticipated from the fact that the $\left(\begin{array}{lll}1 & 10\end{array}\right)$ planes are identical and correspond to a single layer of helices (this is not true of the $(040)$ planes, for example).

It was also possible to detect spherulites of the hexagonal $\beta$-phase in these samples by SAED and their proportion could be increased somewhat by adding a $\beta$-nucleant
Fig. 12 Part of a lath-like region of (1 110$)$ lattice fringes from the film of Fig. 11, apparently containing crystallographic defects (the position of an extra ( 110$)$ half plane is indicated by the arrow)

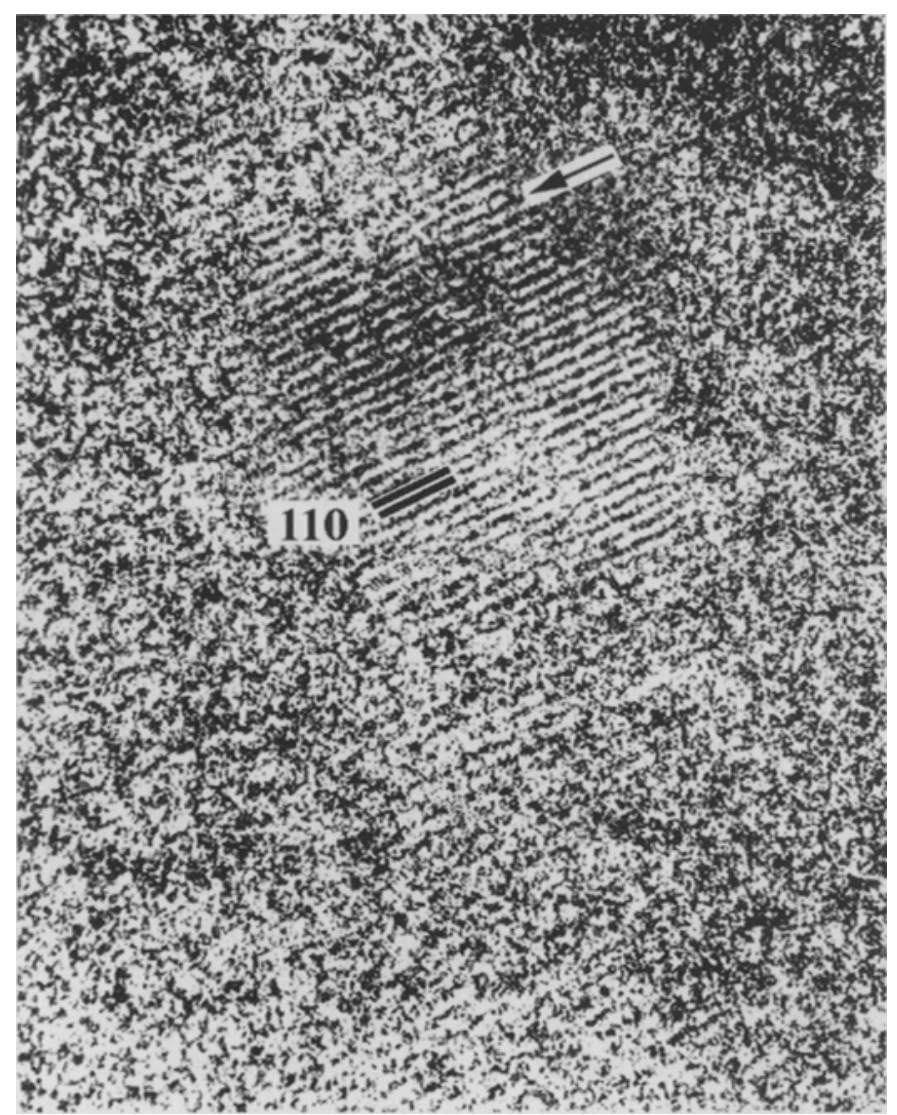


to the solution, although this was less effective for the thinnest films. However, although the $\beta$-lamellae were generally flat-on and their beam sensitivity was comparable with that of the $\alpha$-phase, lattice imaging proved difficult. After several sessions with both the CM 20 the EM 430 ST, using magnifications down to $25000 \times$, we were unable to detect more than one set of lattice fringes in any given region of the HREM images, and this was not pursued further.

Also disappointing were attempts to obtain lattice images from thin $\mathrm{SPP}$ films (the sPP was also supplied by PCD Polymere, Austria). These were melt crystallized on mica at $125^{\circ} \mathrm{C}$, again giving a high proportion of flat-on lamellae. A typical SAED pattern is shown in Fig. 13a, showing characteristic streaking of the $(010)$ and $(210)$ reffections, which has been identified with an intrinsically defective structure $[23,44]$. Briefly, depending on the local handedness of helices being incorporated into the lamellae, the chain packing was proposed to be either fully isochiral or fully antichiral $[23,44]$. In both cases the unit cell is orthorhombic. The nominal parameters for the fully isochiral cell are $a=1.44 \mathrm{~nm}, b=0.56 \mathrm{~nm}, c=0.74$ whereas for the fully antichiral cell $b=1.12 \mathrm{~nm}$, i.e., the unit cell is doubled along the $b$-axis $[23,44]$. Melt crystallized sPP lamellae have been argued to contain a mixture of both modifications, depending on the crystallization temperature, and the electron diffraction patterns have been interpreted in terms of various possible defect arrays [23].

Figure $13 \mathrm{~b}$ shows a typical lattice image of a thin sPP film obtained using the EM 430 ST, showing (200) lattice fringes with a spacing of $0.72 \mathrm{~nm}$. $(010)$ and $(210)$ reflections were absent from most of the image power spectra. This may have been due either to beam damage or noise, or a combination of both. The intensity associated with diffraction from these planes is likely to spread to along the corresponding layer lines so that the local intensity will diminish with respect to the noise level (in view of the earlier comments concerning $\beta$-iPP, it may be significant that the diffraction patterns for $\beta$-iPP also showed streaking; this is possibly linked to local displacements from the expected positions of helices in a perfectly hexagonal array, due to "frustrated" packing [28]).

To gain an idea of what one might hope to observe under the present operating conditions, a rectangular array of alternating rows of helices with a row spacing $(a / 2)$ of $0.72 \mathrm{~nm}$ and a spacing along the rows $(b)$ of $0.56 \mathrm{~nm}$, was constructed using Cerius ${ }^{2}$. Starting at random positions within the array, half rows were shifted by $b / 4$ along their lengths to give a mask corresponding to a possible defect structure shown in Fig. 14a [23] (since we are concerned only with the $c$-axis projection here, no subsequent adjustments were made to the handedness of the helices). For this
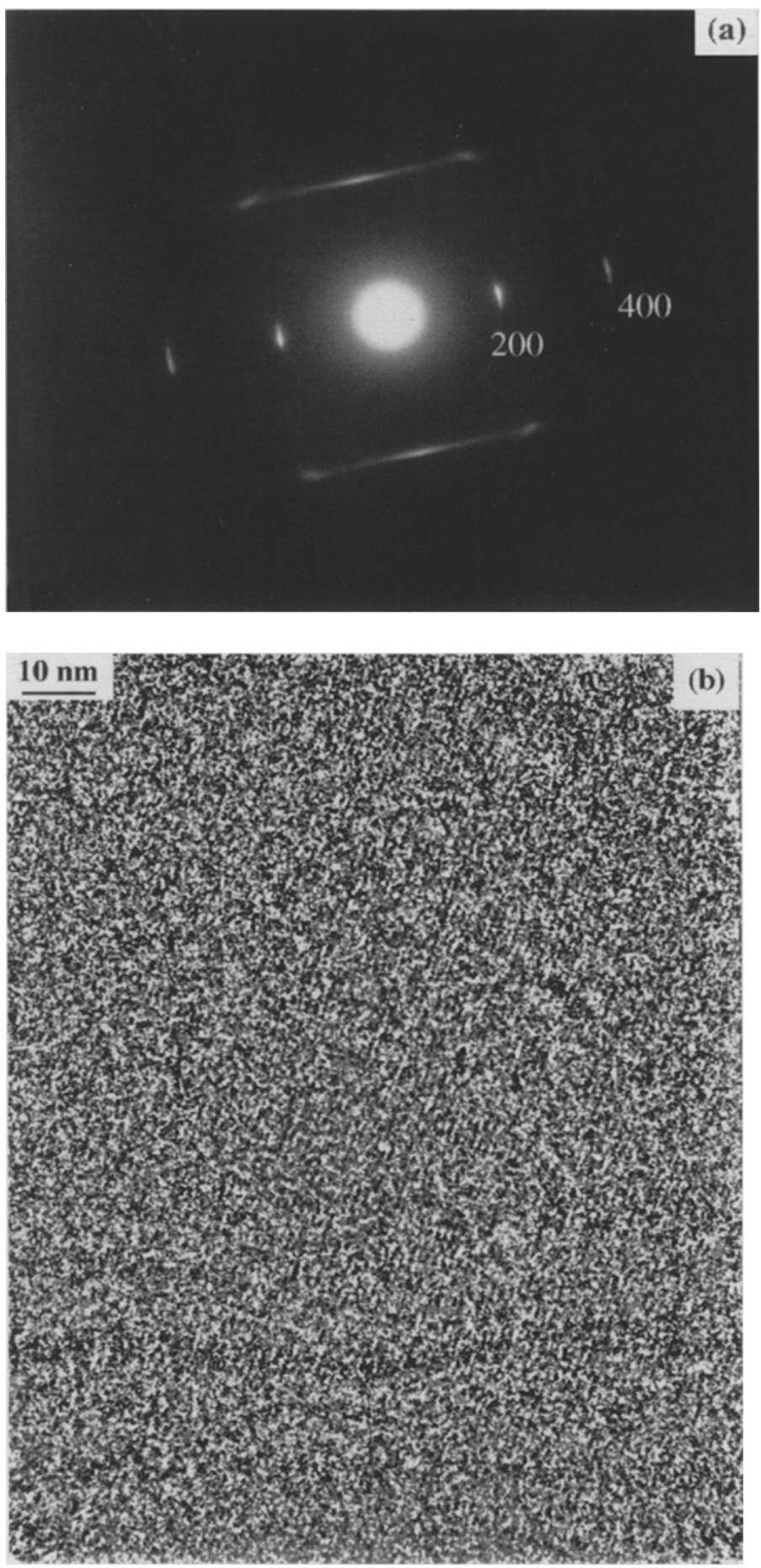

Fig. 13 (a) SAED pattern of sPP melt crystallized at $125^{\circ} \mathrm{C}$; (b) a typical lattice image from the same sample, showing (200) lattice fringes

model structure multislice image calculations were carried out for $t=10 \mathrm{~nm}$ and $\Delta f=-75 \mathrm{~nm}$, and microscope parameters corresponding to the EM 430 ST. Figure 14b shows the simulated image, in which the positions of the 


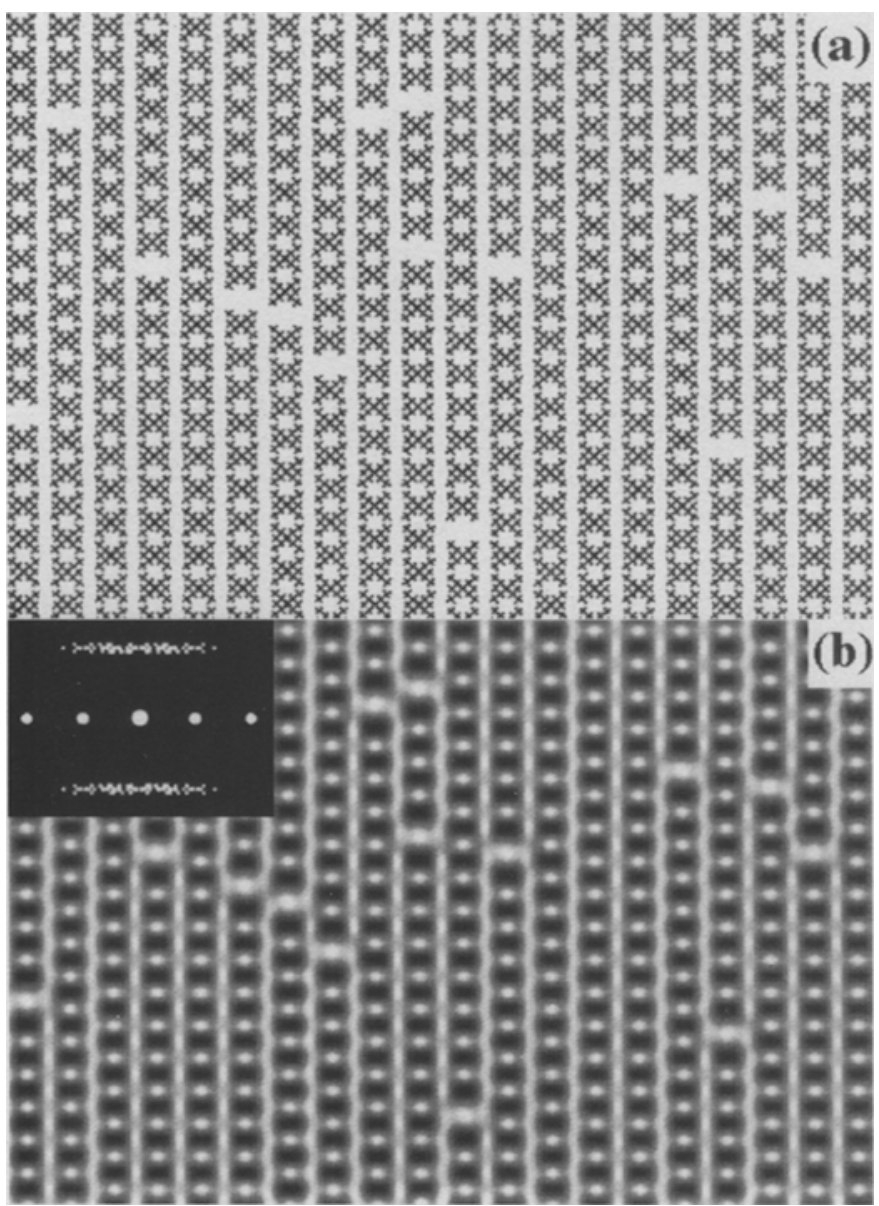

Fig. 14 (a) [0 0101$]$ projection of a model defective sPP lattice; (b) multislice simulation from (a) and the corresponding simulated electron diffraction pattern $(t \sim 10 \mathrm{~nm}, \Delta f=-75 \mathrm{~nm})$

defects are clearly visible, along with the simulated electron diffraction pattern showing streaking of the $(010)$ and (210) peaks, qualitatively consistent with Fig. 13a. When the background noise was included in the simulation by adding the noise component of an experimental lattice image (after filtering out the periodic content), to give a mean signal to noise ratio of $1: 2$, we obtained images such as shown in Fig. 15a. This illustrates the difficulty inherent in detailed interpretation of HREM images of sPP taken under the present conditions. Although the $(200)$ peaks remain clearly identifiable in the power spectrum of this image (Fig. 15b), the intensity of the $(010)$ peaks is reduced, and the associated layer line is substantially lost in the noise. Figure $15 \mathrm{c}$ shows an experimental image at comparable magnification, and its power spectrum (Fig. 15d). Again, the (200) peaks are relatively intense, but the $\left(\begin{array}{lll}0 & 1 & 0\end{array}\right)$ peaks are faint, and the layer lines are apparently absent. It therefore seems unlikely that one
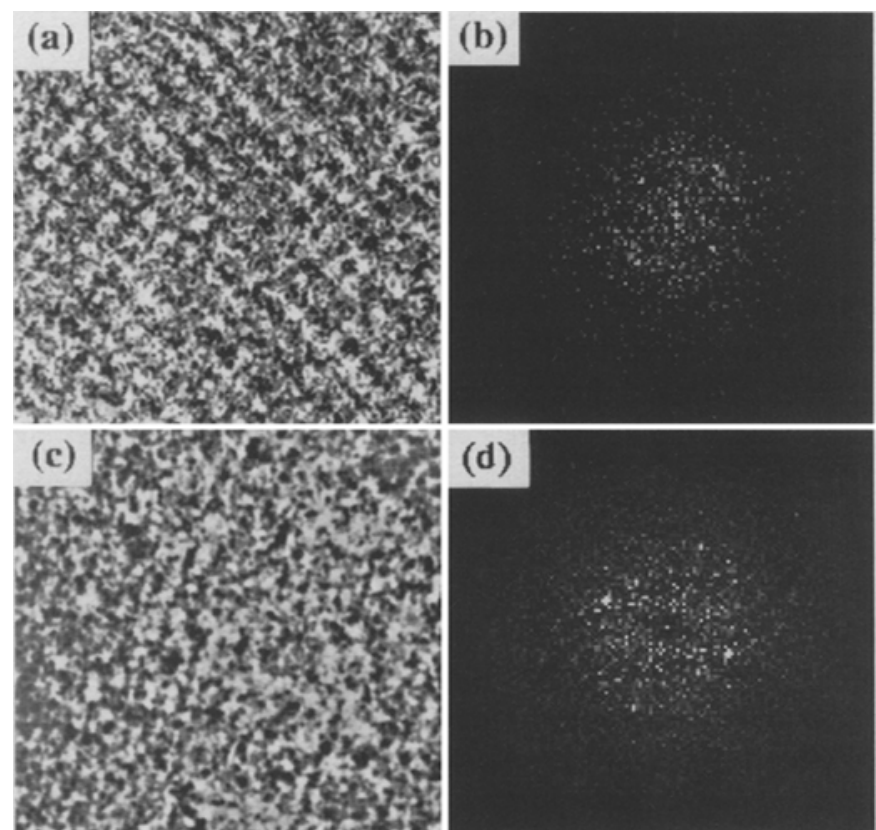

(d)

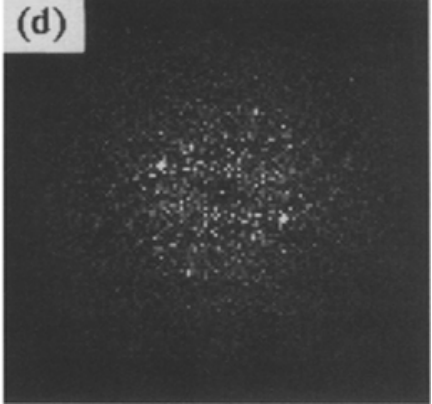

Fig. 15 (a) Multislice image simulation $(t \sim 10 \mathrm{~nm}$ and $\Delta f=-75 \mathrm{~nm}$ ) using the model of Fig. 14, superposed onto the noise component of an experimental image to give a signal to noise ratio $1: 2$; (b) the FFT power spectrum of image (a); (c) experimental image at comparable magnification; (d) the FFT power spectrum of image (c)

can reconstruct the original, presumably defective lattice from images of this quality by simple Fourier filtering.

\section{Conclusions}

Lattice images of the $\alpha$-modification of isotactic polypropylene have been obtained from thin films with spherulitic microstructure using a conventional $200 \mathrm{kV}$ TEM equipped with a low-dose unit, giving a line resolution of up to $0.35 \mathrm{~nm}$. With Fourier filtering, and hence assuming a periodic structure, this should give a comparable effective point resolution as long as the intensities of Bragg peaks in the image power spectrum are higher than the total noise intensity within the masks. However, the noise level becomes more critical for defect structures, in which the Bragg peaks may be both diminished in intensity and smeared out over a wider range of spatial frequencies. Our attempts to obtain HREM images of melt crystallized sPP are a case in point. Even assuming the microscope parameters to be optimized (which the results for $\alpha$-iPP appear to confirm) and leaving aside extrinsic factors such as sample misalignment and beam damage, the effective resolution is severely limited by background noise imposed by the need to underexpose the negatives, the granularity of the film and contributions to the image 
from amorphous material (including the carbon substrate) and lamellae which are not in a Bragg orientation.

It might be of interest to modify the sample preparation conditions in order to reduce the substrate and sample thickness, and to increase the local order (e.g., by crystallizing at higher temperatures). However, well ordered SPP samples have already been extensively characterized by AFM of lamellae crystallized epitaxially from the melt onto soluble substrates [32]. Indeed, whereas TEM is restricted to observation of zones containing strongly diffracting planes, AFM can give access to a much wider range of structural detail in such samples. AFM imaging of defect structures in the [0 011$]$ projection of disordered melt crystallized sPP lamellae nevertheless remains somewhat of a challenge experimentally, not least because of the potentially rough fold surfaces of as-crystallized samples.

Acknowledgments The TEM work was carried out at the Electron Microscopy Center (CIME) of the EPF Lausanne, and we are indebted to Prof. P. Stadelmann and Dr. C. Beeli (CIME) for helpful comments concerning image simulation. We also would like to thank Dr. E. Kramer, Ciba-Geigy, Basic, Switzerland, and Dr. W. Neiß1, PCD Polymere, Linz, Austria, for helpful discussions and samples, and Ciba-Geigy, Switzerland for financial support (RG).

\section{References}

1. Uemura A, Tsuji M, Kawaguchi A, Katayama K (1988) J Mater Sci 23:1506-1509

2. Voigt-Martin IG, Garbella RW, Schumacher M (1992) Macromolecules 25:961-971

3. Fryer JR, Dorset DL (1991) Electron Crystallography of Organic Molecules. Kluwer Academic Publishers, Dordrecht

4. Tsuji M, Kohjiya S (1995) Prog Polym Sci 20:259-308

5. Martin DC, Thomas EL (1995) Polymer 36:1743-1759

6. Revol J-F, John Manley RS (1986) J Mater Sci Lett 5:249-251

7. Chanzy HD, Folda T, Smith P, Gardner K, Revol J-F (1986) J Mater Sci Lett 5:1045-1047

8. Plummer CJG, Kausch H-H (1996) Polymer Bulletin 37:393-397

9. Revol J-F, Chanzy HD, Deslandes Y, Marchessault RH (1989) Polymer 30:1973-1976

10. Revol JF (1985) J Mater Sci Lett 4: 1347-1349

11. Natta G, Corradini P (1960) Del Nuovo Cimento Suppl 15:40-51

12. Turner Jones A, Aizlewood AM, Beckett DR (1964) Makromol Chem 75:134-158

13. Mencik Z (1972) J Macromol Sci-Phys B 6:101-115

14. Hikosaka M, Seto T (1973) Polymer J 5:111-127

15. Immirizi A (1980) Acta Crystallogr B $36: 2378-2385$
16. Corradini P, Petraccone V, Pirozzi B (1983) Eur Polym J 19:299-304

17. Lovinger AJ (1983) J Polym Sci - Polym Phys 21:97-110

18. Guerra G, Petraconne V, Corradini P, De Rosa R, Napolitano R, Pirozzi B, Giunchi G (1984) J Polym Sci - Polym Phys 22:1029-1039

19. De Rosa C, Napolitano RVP, Pirozzi B (1984) Eur Polym J 20:937-941

20. Lotz B, Wittmann JC (1986) J Polym Sci - Polym Phys 24:1541-1558

21. Napolitano R, Pirozzi B, Varriale V (1990) J Polym Sci - Polym Phys 28: 139-147

22. Ferro DR, Brückner S, Meille SV, Ragazzi M (1992) Macromolecules 25: 5231-5235

23. Lovinger AJ, Lotz B, Davis DD, Padden Jr $_{\mathbf{r}}$ FJ (1993) Macromolecules 26: 3494-3503

24. Yan RJ, Li W, Li G, Jiang C (1983) J Macromol Sci - Phys B 32:15

25. Caldas V, Brown GR, Nohr RS, MacDonald JG, Raboin LE (1994) Polymer 35:899-907

26. Meille SV, Ferro DR, Bruckner S, Lovinger AJ, Padden FJ (1994) Macromolecules 27:2615-2622

27. Bu Z et al (1996) Macromolecules 29: 6575-6581

28. Lotz B, Wittmann JC, Lovinger AJ (1996) Polymer 37:4979-4992

29. Lotz B, Wittmann JC, Stocker W, Magonov SN, Cantow HJ (1991) Polym Bull 26:209-214
30. Snetivy D, Guillet JE, Vansco GJ (1993) Polymer 34:429-431

31. Stocker W, Magonov SN, Cantow HJ, Wittmann JC, Lotz B (1993) Macromolecules 26:5915-5923

32. Stocker W, Schumacher M, Graff S, Lang J, Wittmann JC, Lovinger AJ, Lotz B (1994) Macromolecules 27:6948-6955

33. Tsukruk V, Reneker DH (1995) Macromolecules 28:1370-1376

34. Huang MR, Li XG, Fang BR (1995) J Appl Polym Sci 56:1323

35. Lauterwasser BD, Kramer EJ (1979) Phil Mag 39A:469-495

36. Plummer CJG, Kausch H-H (1997) Macromol Chem Phys 198:485-493

37. Montezinos D, Wells BG, Burns JL (1985) J Polym Sci - Polym Lett 23:421

38. Stadelmann PA (1987) Ultramicroscopy 21:131-146

39. Norton DR, Keller A (1985) Polymer 26:704-716

40. Reimer L (1993) Transmission Electron Microscopy. Springer, Berlin

41. Dorset DL (1994) In: Spells SJ (ed) Characterization of Solid Polymers - New Techniques and Developments. Chapman \& Hall, London, pp 1-16

42. Pradère P, Revol JF, John Manley RS (1988) Ultramicroscopy 25:69

43. Martin DC, Thomas EL (1987) MRS Bull XII:27-37

44. Lotz B, Lovinger A, Cais RE (1988) Macromolecules 21:2375-2382 